\title{
Algebraic models for equivariant homotopy theory over Abelian compact Lie groups
}

\author{
Michael A. Mandell ${ }^{1, \star}$, Laura Scull ${ }^{2}$ \\ 1 Department of Mathematics, University of Chicago, Chicago, IL 60637, USA \\ (e-mail: mandell@math.uchicago.edu) \\ 2 Department of Mathematics, The University of Michigan, Ann Arbor, MI 48109, USA \\ (e-mail: laurass@math.lsa.umich.edu)
}

Received: 12 February 2001; in final form: 15 August $2001 /$

Published online: 28 February 2002 - (c) Springer-Verlag 2002

\begin{abstract}
We describe some algebraic models for equivariant rational and $p$-adic homotopy theory over Abelian compact Lie groups.
\end{abstract}

Mathematics Subject Classification (2000):55P91, 55P62, 55P92

\section{Introduction}

Equivariant algebraic topology uses discrete invariants to study spaces with a specified group of symmetries, typically assuming that the symmetry group is both Lie (for actions on manifolds and related spaces) and compact (to make the theory accessible). Since the invariants are discrete, they depend only on homotopy classes of $G$-maps. The domain of equivariant homotopy theory therefore is the homotopy category of $G$-spaces and the goal is to describe this category in algebraic terms. In this paper, we describe a large part of equivariant homotopy theory in algebraic terms when the compact Lie group $G$ is Abelian.

We state our results in the language of localization. A map of $G$-spaces $X \rightarrow Y$ is an equivariant weak equivalence when the induced map on $H$-fixed spaces $X^{H} \rightarrow Y^{H}$ is a (non-equivariant) weak equivalence for every closed subgroup $H \subseteq G$. An equivariant homotopy equivalence is therefore an equivariant weak equivalence, and a fundamental result is that an equivariant weak equivalence is an equivariant homotopy equivalence provided the $G$-spaces are suitably nice, that is, homotopy equivalent to $G$ complexes. Most $G$-spaces of geometric interest (including all $G$-manifolds)

\footnotetext{
* The first author was supported in part by NSF postdoctoral fellowship DMS 9804421.
} 
satisfy this. If we localize the category of $G$-spaces by formally inverting the equivariant weak equivalences, the result is called the equivariant homotopy category.

We can also consider weaker notions of equivalence: A $G$-map $X \rightarrow$ $Y$ is an equivariant rational or $p$-adic equivalence if each induced map $X^{H} \rightarrow Y^{H}$ is a (non-equivariant) rational or $p$-adic equivalence, that is, a homology isomorphism with rational or $\mathbb{Z} / p \mathbb{Z}$ coefficients. The category obtained from $G$-spaces by formally inverting the equivariant rational or $p$-adic equivalences is called the equivariant rational or $p$-adic homotopy category.

The equivariant rational and $p$-adic homotopy categories fracture equivariant homotopy theory into a rational piece and a piece for each prime $p$. By the (non-equivariant) Whitehead theorem, a $G$-map $X \rightarrow Y$ between $G$-spaces with simply connected fixed-point spaces is an equivariant weak equivalence if and only if it is an equivariant rational equivalence and equivariant $p$-adic equivalence for all $p$. Just as in non-equivariant homotopy theory, much of equivariant homotopy theory can be recovered from rational homotopy theory, $p$-adic homotopy theory, and patching information.

We describe algebraic models for rational and $p$-adic equivariant homotopy theory. In the rational case, our description is in terms of diagrams of commutative differential graded $\mathbb{Q}$-algebras (CDGA's) of a particular shape. In Sect. 2 , we describe a category $\mathcal{D}$ closely related to the lattice of closed subgroups of $G$. We define a $\mathcal{D}$-CDGA to be a functor from $\mathcal{D}$ into the category of commutative differential graded $\mathbb{Q}$-algebras. We define a contravariant functor $\underline{\mathrm{A}}_{0}$ from the category of $G$-spaces to the category of $\mathcal{D}$-CDGAs; the value at each object of $\mathcal{D}$ is the Thom-Sullivan De Rham algebra of the singular complex of a Borel construction on a fixed point space of the given $G$-space; see Sect. 3 for details. We let $\underline{P}_{0}$ be a cofibrant approximation of $\underline{\mathrm{A}}_{0}(*)$, where $*$ is the one-point $G$-space; essentially, this is a minimal model for $\underline{\mathrm{A}}_{0}(*)$. Then we can regard $\underline{\mathrm{A}}_{0}$ as a functor from $G$ spaces to the category of $\mathcal{D}$-CDGAs under $\underline{P}_{0}$. We will see that $\underline{\mathrm{A}}_{0}$ converts equivariant rational equivalences to (objectwise) quasi-isomorphisms, and so $\underline{A}_{0}$ passes to a functor on the homotopy categories. In the rational case, our main result is the following.

Theorem A. Let $G$ be an Abelian compact Lie group. There is a functor $\underline{A}_{0}$ from the equivariant rational homotopy category to the homotopy category of $\mathcal{D}$-CDGAs under $\underline{P}_{0}$. On the full subcategory of $G$-simply connected $G$-finite $\mathbb{Q}$-type $G$-spaces, this functor is full and faithful.

In the $p$-adic case, we use $E_{\infty} \overline{\mathbf{F}}_{p}$-algebras in place of rational differential graded $\mathbb{Q}$-algebras, and the cochain complex in place of the De Rham complex, but otherwise essentially the same outline holds. We prove the following theorem. 
Theorem B. Let $G$ be an Abelian compact Lie group. There is a functor $\underline{A}_{p}$ from the equivariant $p$-adic homotopy category to the homotopy category of $\mathcal{D}$ - $E_{\infty} D G A s$ under $\underline{P}_{p}$. On the full subcategory of $G$-simply connected $G$-finite p-type $G$-spaces, this functor is full and faithful.

Here $G$-simply connected means that each fixed-point space is simply connected in the non-equivariant sense (and may be empty or nonconnected). Likewise, $G$-finite $\mathbb{Q}$ - or $p$ - type means that each fixed-point space is (non-equivariantly) finite $\mathbb{Q}$ - or $p$ - type, that is, its homology with coefficients in $\mathbb{Q}$ or $\mathbb{Z} / p \mathbb{Z}$ is finitely generated in each degree. In the case of each of these theorems, we can describe in homological terms the image of the $G$-simply connected $G$-finite type $G$-spaces; see Sect. 3 for details.

Unfortunately, we are less successful at describing the objects $\underline{P}_{0}$ and $\underline{P}_{p}$, and so the models obtained from our main theorems are not very explicit in general. When $\operatorname{dim} G=0$, that is, when $G$ is a finite group, we can take $\underline{P}_{0}$ to be the constant diagram on $\mathbb{Q}$ in the rational case and $\underline{P}_{p}$ to be the constant diagram on $\overline{\mathbf{F}}_{p}$ in the $p$-adic case. The models we obtain this way are more complicated than the ones described in [11] (rationally) and in [7] ( $p$-adically), where the methods apply more generally to non-Abelian finite groups. In the case when $G$ is the circle group $\mathbb{T}$, we can also describe our rational models explicitly; we do this in Sect. 4. This is the algebraic category described in the second author's 1999 University of Chicago thesis [9].

\section{Diagrams of spaces}

In order to produce algebraic models, we first reduce equivariant homotopy theory to the study of diagrams of spaces and fibrations, and then apply the algebraic models of [10] and [6]. Historically the first description of equivariant homotopy theory in terms of diagrams is the theorem of Elmendorf [5] that explains how to recover a $G$-space $X$ up to equivariant weak equivalence from its system of fixed-point spaces $X^{H}$ and inclusion relations.

To make this precise, consider the orbit category $\mathcal{O}_{G}$ of $G$, whose objects are the canonical orbits $G / H$ for all closed subgroups $H \subseteq G$, and whose maps are equivariant maps between them. Any map in $\mathcal{O}_{G}(G / H, G / K)$ is of the form $g H \rightarrow g a K$ for some $a \in G$ with such that $a^{-1} H a \subseteq K$, and moreover that $a$ and $b$ represent the same map if and only if $a b^{-1} \in K$. So the maps in this category are given by

$$
\mathcal{O}_{G}(G / H, G / K) \cong(G / K)^{H} .
$$

We regard $\mathcal{O}_{G}$ as a topological category, topologizing the maps by the above isomorphism. 
Associated to any $G$-space $X$ there is a contravariant fixed point functor $\Phi X$ from $\mathcal{O}_{G}$ to spaces. This functor has the value $\Phi X(G / H)=X^{H}$, with morphisms induced by the $G$-action on the space $X$. This functor is continuous in the sense that the map

$$
\mathcal{O}_{G}(G / K, G / H) \times \Phi X(G / H) \rightarrow \Phi X(G / K)
$$

is a continuous map for every pair of objects $G / K, G / H$ of $\mathcal{O}_{G}$. Motivated by this example we define the category $\mathcal{O}_{G} \mathcal{U}$ of $\mathcal{O}_{G}$-spaces to be the category of continuous contravariant functors from $\mathcal{O}_{G}$ to spaces. We define weak equivalence, rational equivalence, and $p$-adic equivalence in $\mathcal{O}_{G} \mathcal{U}$ objectwise; we say that a map $\underline{X} \rightarrow \underline{Y}$ in $\mathcal{O}_{G} \mathcal{U}$ is a weak (resp. rational, p-adic) equivalence if $\underline{X}(G / H) \rightarrow \underline{Y}(G / H)$ is a non-equivariant weak (resp. rational, $p$-adic) equivalence for every object $G / H$ of $\mathcal{O}_{G}$. We define the homotopy category, the rational homotopy category, and the $p$-adic homotopy category of $\mathcal{O}_{G}$-spaces by formally inverting the weak equivalences, rational equivalences, and $p$-adic equivalences respectively.

We can interpret $\Phi$ as a functor from the category of $G$-spaces to $\mathcal{O}_{G} \mathcal{U}$. The earlier definitions immediately imply that a map of $G$-spaces $X \rightarrow Y$ is an equivariant weak, rational, or $p$-adic equivalence if and only if the induced map $\Phi X \rightarrow \Phi Y$ is a weak, rational, or $p$-adic equivalence in $\mathcal{O}_{G} \mathcal{U}$ respectively. It follows that $\Phi$ factors through the homotopy categories we obtain by inverting these equivalences. The following result shows that $\Phi X$ contains exactly the same homotopy information as the original space $X$.

Theorem 1.1. (Elmendorf) The functor $\Phi$ induces an equivalence of:

(i) The equivariant homotopy category and the homotopy category of $\mathcal{O}_{G^{-}}$ spaces,

(ii) The equivariant rational homotopy category and the rational homotopy category of $\mathcal{O}_{G}$-spaces, and

(iii) The equivariant p-adic homotopy category and the p-adic homotopy category of $\mathcal{O}_{G}$-spaces for each prime $p$.

Proof. Elmendorf [5] constructs a functor $C$ from $\mathcal{O}_{G}$-spaces to $G$-spaces, and natural weak equivalences $C \Phi \rightarrow \mathrm{Id}$ and $\Phi C \rightarrow \mathrm{Id}$.

For reasons explained in the next section, we find it convenient to work with a variant of the orbit category that we call the subdivided orbit category $\Delta \mathcal{O}_{G}$. Although Elmendorf's theorem holds very generally, here we need to restrict to the case when $G$ is Abelian. In this case, the orbit $G / K$ has an action by the group $G / K$. The idea for the category $\Delta \mathcal{O}_{G}$ is to have many objects corresponding to each orbit $G / K$, one for each group $G / H$ that can act on it. We use the symbol $G / K[H]$ to denote the orbit $G / K$ thought of as a $G / H$ space. Following this idea, for maps, we 
have $\Delta \mathcal{O}_{G}(G / K[H], G / K[H])=G / H$. As $K$ varies, we have a canonical quotient map $G / K^{\prime}[H] \rightarrow G / K[H]$ whenever $K^{\prime} \subseteq K$. In addition, as $H$ varies, we have a canonical group-change map $G / K[H] \rightarrow$ $G / K\left[H^{\prime}\right]$ whenever $H^{\prime} \subseteq H$. To understand the variance in $H$ (which may appear backwards), observe that for a contravariant functor $X$, the map $X\left(G / K\left[H^{\prime}\right]\right) \rightarrow X(G / K[H])$ goes from a space with some group acting to a space with a quotient group acting. This leads to the precise definition of the category $\Delta \mathcal{O}_{G}$. We emphasize that the variance in $H$ is opposite to that of $K$.

Definition 1.2. Let $\Delta \mathcal{O}_{G}$ be the category that has one object $G / K[H]$ for each pair of closed subgroups $H \subseteq K$. Maps are defined by

$$
\Delta \mathcal{O}_{G}\left(G / K_{1}\left[H_{1}\right], G / K_{2}\left[H_{2}\right]\right)= \begin{cases}G / H_{1} & \text { if } H_{2} \subseteq H_{1} \subseteq K_{1} \subseteq K_{2} \\ \emptyset & \text { otherwise }\end{cases}
$$

Composition of maps is induced by multiplication in $G$. We define the category $\Delta \mathcal{O}_{G} \mathcal{U}$ of $\Delta \mathcal{O}_{G}$-spaces to be the category of continuous contravariant functors from $\Delta \mathcal{O}_{G}$ to spaces.

Again we define weak equivalences, rational equivalences, and $p$-adic equivalences objectwise, and form the homotopy category, rational homotopy category, and $p$-adic homotopy category of $\Delta \mathcal{O}_{G}$-spaces by formally inverting the corresponding equivalences.

Regarding the object $G / K[H]$ as the orbit $G / K$ with a $G / H$-action defines a (covariant) functor from $\Delta \mathcal{O}_{G}$ to $\mathcal{O}_{G}$ : The functor sends the object $G / K[H]$ to $G / K$, and sends the morphism space $\Delta \mathcal{O}_{G}\left(G / K_{1}\left[H_{1}\right], G / K_{2}\left[H_{2}\right]\right)=$ $G / H_{1}$ to the morphism space $\mathcal{O}_{G}\left(G / K_{1}, G / K_{2}\right)=G / K_{2}$ via the quotient map when $H_{2} \subseteq H_{1} \subseteq K_{1} \subseteq K_{2}$. Composing with the functor $\Delta \mathcal{O}_{G} \rightarrow \mathcal{O}_{G}$, we therefore obtain a functor $I$ from $\mathcal{O}_{G} \mathcal{U}$ to $\Delta \mathcal{O}_{G} \mathcal{U}$. Clearly $I$ preserves weak equivalences, rational equivalences, and $p$-adic equivalences, and so it passes to the categories obtained by formally inverting these equivalences. In Sect. 7, we show:

Theorem 1.3. The functor I induces full embeddings of:

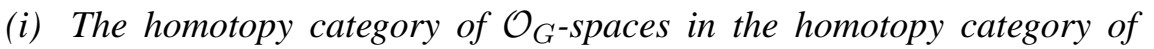
$\Delta \mathcal{O}_{G}$-spaces,

(ii) The rational homotopy category of $\mathcal{O}_{G}$-spaces in the rational homotopy category of $\Delta \mathcal{O}_{G}$-spaces, and

(iii) The p-adic homotopy category of $\mathcal{O}_{G}$-spaces in the p-adic homotopy category of $\Delta \mathcal{O}_{G}$-spaces for each prime $p$.

In order to understand the embeddings, we also want to identify the image of $I$. If we start with an object $\underline{X}$ in $\mathcal{O}_{G} \mathcal{U}$, then by definition $I \underline{X}(G / K[H])=$ 
$\underline{X}(G / K)$ for every $H \subseteq K$. Define the category $\operatorname{Im}^{\Delta}$ to be the full subcategory of the homotopy category of $\Delta \mathcal{O}_{G} \mathcal{U}$ consisting of those objects for which any map $\underline{X}(G / K[H]) \rightarrow \underline{X}(G / K[K])$ is a weak equivalence for all subgroups $H \subseteq K$; likewise define $\operatorname{Im}_{0}^{\Delta}$ and $\operatorname{Im}_{p}^{\Delta}$ to be the respective full subcategories of the rational and $p$-adic homotopy categories of $\Delta \mathcal{O}_{G} \mathcal{U}$ consisting of those objects for which any map $\underline{X}(G / K[H]) \rightarrow \underline{X}(G / K[K])$ is a rational and $p$-adic equivalence. In Sect. 7 , we prove:

Theorem 1.4. An object $\underline{X}$ in the homotopy category, rational homotopy category, or p-adic homotopy category of $\Delta \mathcal{O}_{G}$-spaces is isomorphic to an object in the image of $I$ if and only if $X$ is in $\operatorname{Im}^{\Delta}, \operatorname{Im}_{0}^{\Delta}$, or $\operatorname{Im}_{p}^{\Delta}$ respectively.

Thus, we have equivalences of categories between the equivariant homotopy category and $\mathrm{Im}^{\Delta}$, the equivariant rational homotopy category and $I m_{0}^{\Delta}$, and the equivariant $p$-adic homotopy category and $I m_{p}^{\Delta}$.

\section{Diagrams of bundles}

Since the categories $\mathcal{O}_{G}$ and $\Delta \mathcal{O}_{G}$ are topologized, $\mathcal{O}_{G^{-}}$-spaces and $\Delta \mathcal{O}_{G^{-}}$ spaces cannot be used directly to produce algebraic models unless $G$ is finite. Dwyer and Kan [3] showed that equivariant homotopy theory can be reduced to the theory of diagrams on a certain discrete category and the theory of fibrations. In this section, we describe a slightly different such reduction that follows similar ideas.

We say that a map of $G$-spaces $X \rightarrow Y$ is an underlying weak equivalence when it is a non-equivariant weak equivalence. The projection map $E G \times X \rightarrow X$ is an underlying weak equivalence, and so every $G$-space is underlying weak equivalent to a free $G$-space. A basic tenet of bundle theory is that a $G$-space $X$ is determined up to underlying weak equivalence by the classifying map $q: E G \times_{G} X \rightarrow B G$. Precisely, $X$ is underlying weak equivalent to the $G$-space obtained as the pullback along $q$ of the map $E G \rightarrow B G$, as this is again the $G$-space $E G \times X$. In an $\mathcal{O}_{G}$-space $\underline{X}$, the $G / H$-space $\underline{X}(G / H)$ is determined only up to underlying weak equivalence, and this piece of the structure can therefore be recovered from a classifying map. The goal is to fit these classifying maps together in such a way that the whole $\mathcal{O}_{G}$-space can be recovered.

The first difficulty is that the different objects in the $\mathcal{O}_{G}$ diagram have actions of different groups. For an Abelian group $G$, the universal bundle $E(G / H) \rightarrow B(G / H)$ is covariant in $G / H$, whereas the spaces $X(G / H)$ in an $\mathcal{O}_{G}$-space $\underline{X}$ are contravariant. We have introduced the subdivided orbit category $\Delta \mathcal{O}_{G}$ in the previous section precisely to deal with this problem. 
For a map $G / K_{1}\left[H_{1}\right] \rightarrow G / K_{2}\left[H_{2}\right]$, we have contravariance both in the universal bundles

$$
\left(E\left(G / H_{1}\right) \rightarrow B\left(G / H_{1}\right)\right) \longleftarrow\left(E\left(G / H_{2}\right) \rightarrow B\left(G / H_{2}\right)\right)
$$

and the spaces $\underline{X}\left(G / K_{1}\left[H_{1}\right]\right) \leftarrow \underline{X}\left(G / K_{2}\left[H_{2}\right]\right)$. In fact, in contrast to $\mathcal{O}_{G}$-spaces, we can define a $\Delta \mathcal{O}_{G}$-space $\underline{E}$ by

$$
\underline{E}(G / K[H])=E(G / H) .
$$

The final map $\underline{E} \rightarrow \underline{*}$ is a weak equivalence.

Before proceeding, we should note one other complication. We can recover the $G$-space $X$ up to underlying weak equivalence from the classifying map $E G \times{ }_{G} X \rightarrow B G$, even if it is known only up to weak equivalence in the category of spaces over $B G$. However, if we know the classifying map only up to rational or $p$-adic equivalence, we cannot necessarily recover $X$ up to underlying rational or $p$-adic equivalence. An instructive example is the case of $X=S^{2}$ with the antipodal action of $G=\mathbb{Z} / 2 \mathbb{Z}$. Here, the map $S^{2} \rightarrow *$ is not a rational equivalence but induces a rational equivalence on Borel constructions. The classifying map therefore cannot distinguish the free $\mathbb{Z} / 2 \mathbb{Z}$-space $S^{2}$ from the trivial $\mathbb{Z} / 2 \mathbb{Z}$-space $*$. This problem is directly related to the fact that the group in the example is not connected. When $G$ is connected, $B G$ is simply connected, and it follows from the ideas of Eilenberg and Moore [4] that pullbacks along the fibration $E G \rightarrow B G$ preserve homology isomorphisms. For this reason, we base our main bundle constructions on an action of the identity component $G_{e}$ and separate out the action of the finite group $\pi_{0} G=G / G_{e}$. Instead of working with the classifying space $B G$, we work instead with the $\pi_{0} G$-space $E G / G_{e}$, which is (non-equivariantly) equivalent to $B\left(G_{e}\right)$.

Now we combine these two ideas to define a category $\mathcal{B}$ of bundle diagrams whose various homotopy categories are equivalent to the corresponding homotopy categories of $\Delta \mathcal{O}_{G}$-spaces. Motivated by the observations above, the functor from $\Delta \mathcal{O}_{G}$-spaces to this new category takes $\underline{X}$ to a diagram of bundles of the form

$$
E(G / H) \times{ }_{(G / H)_{e}} \underline{X}(G / K[H]) \rightarrow E(G / H) /(G / H)_{e},
$$

in other words, of the form

$$
\underline{E}(G / K[H]) \times(G / H)_{e} \underline{X}(G / K[H]) \rightarrow \underline{E}(G / K[H]) \times(G / H)_{e} * .
$$

We want our diagrams to be indexed on a discrete category $\mathcal{D}$, and examining how these bundles fit together leads us to the following definition. 
Definition 2.2. Let $\mathcal{D}$ be the homotopy category of $\Delta \mathcal{O}_{G}$ : its objects are the same as the objects of $\Delta \mathcal{O}_{G}$, and maps are

$$
\begin{aligned}
\mathcal{D}\left(G / K_{1}\left[H_{1}\right], G / K_{2}\left[H_{2}\right]\right) & =\pi_{0}\left(\Delta \mathcal{O}_{G}\left(G / K_{1}\left[H_{1}\right], G / K_{2}\left[H_{2}\right]\right)\right) \\
& =\pi_{0}\left(G / H_{1}\right)
\end{aligned}
$$

if $H_{2} \subseteq H_{1} \subseteq K_{1} \subseteq K_{2}$ and $\emptyset$ otherwise. We define the category $\mathcal{D U}$ of $\mathcal{D}$-spaces to be the category of contravariant functors from $\mathcal{D}$ to spaces.

We emphasize that although $\mathcal{D}$ is a homotopy category, a functor in $\mathcal{D U}$ has codomain the category of spaces as opposed to the homotopy category of spaces.

The prescription

$$
Q \underline{X}(G / K[H])=\underline{E}(G / K[H]) \times{ }_{(G / H)_{e}} \underline{X}(G / K[H])
$$

defines a functor $Q$ from $\Delta \mathcal{O}_{G}$-spaces to $\mathcal{D}$-spaces. We write $\underline{B}$ for $Q \underline{\underline{*}}$, where $*$ is the constant $\Delta \mathcal{O}_{G}$-space on the one-point space. We regard $Q$ as a functor from $\mathcal{O}_{G} \mathcal{U}$ to $\mathcal{D U} / \underline{B}$.

Definition 2.3. The category $\mathcal{B}$ is the category $\mathcal{D U} / \underline{B}$ of $\mathcal{D}$-spaces lying over $\underline{B}$.

In other words, an object of $\mathcal{B}$ consists of an object $\underline{Y}$ of $\mathcal{D U}$ together with a map $\underline{Y} \rightarrow \underline{B}$. A map in $\mathcal{B}$ is a map in $\mathcal{D U}$ that commutes with the maps to $\underline{B}$.

We define weak equivalences, rational equivalences, and $p$-adic equivalences in $\mathcal{B}$ objectwise for the underlying $\mathcal{D}$-space. As always, we form the homotopy category, rational homotopy category, and $p$-adic homotopy category of $\mathcal{B}$ by formally inverting those maps that are weak equivalences, rational equivalences, and $p$-adic equivalences respectively. It follows from classical bundle theory (and the Serre spectral sequence) that the functor $Q$ preserves weak equivalences, rational equivalences, and $p$-adic equivalences, and so we obtain induced functors between the various homotopy categories of $\Delta \mathcal{O}_{G}$-spaces and the corresponding homotopy categories of $\mathcal{B}$. In Sect. 6, we show:

Theorem 2.4. The functor $Q$ induces equivalences between the homotopy category, rational homotopy category and p-adic homotopy category of $\Delta \mathcal{O}_{G}$-spaces and the corresponding homotopy categories of $\mathcal{B}$.

Once again, we also wish to identify those objects equivalent to $G$-spaces, or equivalently, to $\mathcal{O}_{G}$-spaces. For an $\mathcal{O}_{G}$-space $\underline{X}$, the $\Delta \mathcal{O}_{G}$-space $I \underline{X}$ has the property that

$$
I \underline{X}(G / K[H])=\underline{X}(G / K)=I \underline{X}(G / K[K])
$$


and the structure maps $G / K[K] \rightarrow G / K[H]$ induce the identity isomorphism. It follows that for the object $Q I \underline{X}$ of $\mathcal{B}$,

$$
\begin{aligned}
Q I \underline{X}(G / K[H]) & =E(G / H) \times_{(G / H)_{e}} I \underline{X}(G / K[H]) \\
& =E(G / H) \times_{(G / H)_{e}} \underline{X}(G / K) \\
& \cong B(G / H) \times_{B(G / K)} E(G / K) \times_{(G / K)_{e}} \underline{X}(G / K) \\
& \cong \underline{B}(G / K[H]) \times_{\underline{B}(G / K[K])} Q I \underline{X}(G / K[K]) .
\end{aligned}
$$

With this as motivation, we define $I m^{\mathcal{B}}, \operatorname{Im}_{0}^{\mathcal{B}}$, and $I m_{p}^{\mathcal{B}}$ to be the full subcategories of the homotopy category, rational homotopy category, and $p$-adic homotopy category of $\mathcal{B}$, consisting of those objects $\underline{Y}$ for which the map

$$
\underline{Y}(G / K[H]) \rightarrow \underline{B}(G / K[H]) \times_{\underline{B}(G / K[K])} \underline{Y}(G / K[K]) .
$$

induced by $[e] \in \pi_{0} G / K=\mathcal{D}(G / K[K], G / K[H])$ is a weak equivalence, rational equivalence, and $p$-adic equivalence respectively. In Sect. 6, we prove:

Theorem 2.5. An object $\underline{Y}$ in the homotopy category, rational homotopy category, or p-adic homotopy category of $\mathcal{B}$ is isomorphic to an object in the image of $Q I$ if and only if $X$ is in $\operatorname{Im}^{\mathcal{B}}, \operatorname{Im}_{0}^{\mathcal{B}}$, or $\operatorname{Im}_{p}^{\mathcal{B}}$ respectively.

\section{The algebraization theorems}

Armed with descriptions of the equivariant rational and $p$-adic homotopy categories in terms of diagrams on a discrete category, we explain the algebraization theorems. The basic idea is that any (contravariant) functor from spaces to some category of algebras defines a functor from the over category $\mathcal{B}$ to the category of (covariant) $\mathcal{D}$-diagrams of algebras lying under the diagram obtained from $\underline{B}$. When the functor preserves rational or $\mathbb{Z} / p \mathbb{Z}$ homology isomorphisms, it induces a functor on the rational or $p$-adic homotopy categories. We apply this observation to the Thom-Sullivan De Rham and singular cochain functors.

Definition 3.1. Let $\Omega^{*}$ denote the functor from spaces to commutative differential graded $\mathbb{Q}$-algebras obtained by applying the polynomial De Rham functor of [10] to the singular simplicial set of a space. Let $C^{*}$ denote the singular cochain functor from spaces to $E_{\infty} \overline{\mathbf{F}}_{p}$-algebras.

We consider the category of (covariant) functors from $\mathcal{D}$ to commutative differential graded $\mathbb{Q}$-algebras and likewise to $E_{\infty} \overline{\mathbf{F}}_{p}$-algebras. We call 
objects in these categories $\mathcal{D}$-CDGAs and $\mathcal{D}$ - $E_{\infty}$ DGAs respectively. Weak equivalences in these categories are maps that are quasi-isomorphisms at each object. Applying the functors $\Omega^{*}$ and $C^{*}$ objectwise to a $\mathcal{D}$-space, we obtain functors from $\mathcal{D}$-spaces to $\mathcal{D}$-algebras.

Definition 3.2. Let $\Omega^{*}$ be the functor from $\mathcal{D}$-spaces to $\mathcal{D}$-CDGAs defined by applying $\Omega^{*}$ objectwise. Let $\underline{C}^{*}$ be the functor from $\mathcal{D}$-spaces to $\mathcal{D}$ $E_{\infty} D G A s$ defined by applying $C^{*}$ objectwise.

If we apply the functor $\underline{\Omega}^{*}$ to an object $\underline{Y}$ of $\mathcal{B}$, we obtain not just a $\mathcal{D}$ CDGA $\underline{\Omega}^{*} \underline{Y}$, but also a map of $\mathcal{D}$-CDGAs $\underline{\Omega}^{*} \underline{B} \rightarrow \underline{\Omega}^{*} \underline{Y}$. Since the structure map $\underline{Y} \rightarrow \underline{B}$ is necessary in the definition of the category $\mathcal{B}$ to obtain the embedding of the rational equivariant homotopy category, we should expect that to obtain an embedding of the rational homotopy category into a homotopy category in algebra, we should need some form of the structure map $\underline{\Omega}^{*} \underline{B} \rightarrow \underline{\Omega}^{*} \underline{Y}$. We could look at the category of $\mathcal{D}$-CDGAs under $\Omega^{*} \underline{B}$, but this might not have the correct homotopy category. An analogy to keep in mind is that the category of spaces under a given space $X$ typically does not contain all the homotopy types expected from the homotopy type of $X$ if $X$ is not homotopy equivalent to a CW complex. Similarly, we need to look at the category of $\mathcal{D}$-CDGAs under a suitably nice quasi-isomorphic replacement for $\underline{\Omega}^{*} \underline{B}$.

In [7] it is shown that a diagram category of commutative differential graded $\mathbb{Q}$-algebras or of $E_{\infty} \overline{\mathbf{F}}_{p}$-algebras is a closed model category. We therefore have a notion of cofibrant object, and the factoring axioms allow us to choose a cofibrant approximation $\underline{P}_{0}$ of $\underline{\Omega}^{*} \underline{B}$ and a cofibrant approximation $\underline{P}_{p}$ of $\underline{C}^{*} \underline{B}$. This is one precise meaning for the phrase "suitably nice quasi-isomorphic replacement". However, to prove the theorems we are after, we do not need to put such a stringent requirement on $\underline{P}_{0}$ and $\underline{P}_{p}$; we only need to choose an objectwise cofibrant approximation. Since we assume that the reader interested in the $p$-adic case is familiar with [6], we do not review the definition of a cofibrant $E_{\infty} \overline{\mathbf{F}}_{p}$-algebra. It is entirely analogous to the definition of a cofibrant commutative differential graded $\mathbb{Q}$-algebra from [1], which we do review.

Definition 3.3. Let $R$ be a commutative differential graded $\mathbb{Q}$-algebra (CDGA). We say that $R$ is cellular if there exist graded submodules $\left(X_{0}=0\right)$, $X_{1}, X_{2}, \ldots$ of the underlying graded module of $R$ such that

(i) The differential of any element in $X_{n}$ is in the sub-graded algebra generated by $X_{0}, \ldots, X_{n-1}$.

(ii) The underlying graded algebra of $R$ is the free graded commutative algebra on $X_{1} \oplus X_{2} \oplus \cdots$.

We say that $R$ is cofibrant if it is a retract of a cellular CDGA. 
A standard trick in model category theory for finding a weak equivalence from a cellular object to an arbitrary object is called Quillen's small object argument, and it is explained for CDGA's in [1, pp. 20-22]. In this context, it gives a functor $\Gamma$ from the category of CDGA's to itself and a natural quasi-isomorphism $\Gamma \rightarrow \mathrm{Id}$ such that for any CDGA $R, \Gamma R$ is a cellular CDGA. We have an analogous construction for $E_{\infty}$ DGA's. The following proposition is an immediate consequence.

Proposition 3.4. There exists a D-CDGA $\underline{P}_{0}$ and a quasi-isomorphism $\underline{P}_{0} \rightarrow \underline{\Omega}^{*} \underline{B}$, such that $\underline{P}_{0}(G / K[H])$ is a cofibrant $C D G A$ for every $G / K[H]$ in $\mathcal{D}$. There exists a $\mathcal{D}-E_{\infty} D G A \underline{P}_{p}$ and a quasi-isomorphism $\underline{P}_{p} \rightarrow \underline{C}^{*} \underline{B}$, such that $\underline{P}_{p}(G / K[H])$ is a cofibrant $E_{\infty} D G A$ for every $G / K[H]$ in $\mathcal{D}$.

We choose and fix such objects $\underline{P}_{0}$ and $\underline{P}_{p}$. The functors $\underline{\Omega}^{*}$ and $\underline{C}^{*}$ now take objects in $\mathcal{B}$ to objects under $\underline{P}_{0}$ and $\underline{P}_{p}$. We denote these undercategories as $\mathcal{A}_{0}$ and $\mathcal{A}_{p}$ respectively.

Definition 3.5. Let $\mathrm{A}_{0}$ denote the functor from $\mathcal{B}$ to $\mathcal{A}_{0}$ induced by $\underline{\Omega}^{*}$; let $\mathrm{A}_{p}$ denote the functor from $\mathcal{B}$ to $\mathcal{A}_{p}$ induced by $\underline{C}^{*}$. We define the functor $\underline{\mathrm{A}}_{0}$ from $G$-spaces to $\mathcal{A}_{0}$ to be the composite $\mathrm{A}_{0} \circ Q \circ I \circ \Phi$, and the functor $\underline{\mathrm{A}}_{p}$ from $G$-spaces to $\mathcal{A}_{p}$ to be the composite $\mathrm{A}_{p} \circ Q \circ I \circ \Phi$.

The functor $\mathrm{A}_{0}$ converts rational equivalences to quasi-isomorphisms and the functor $\mathrm{A}_{p}$ converts $p$-adic equivalences to quasi-isomorphisms. We therefore obtain functors $A_{0}$ and $A_{p}$ on the homotopy categories obtained by inverting these equivalences.

We can now explain the main theorems of the introduction. We say that an object $\underline{Y}$ of $\mathcal{B}$ is simply connected if each component of $\underline{Y}(G / K[H])$ is simply connected for all $G / K[H]$. Likewise, we say that $\underline{Y}$ is finite $\mathbb{Q}$ or $p$ - type if each $\underline{Y}(G / K[H])$ is finite $\mathbb{Q}$ - or $p$ - type. Clearly, when a $G$ space $X$ is $G$-simply connected and $G$-finite type, the object $Q I \Phi X$ of $\mathcal{B}$ is simply connected and finite type. Theorems 1.1, 1.3, and 2.4 show that the functor $Q \circ I \circ \Phi$ embeds the rational equivariant homotopy category in the rational homotopy category of $\mathcal{B}$ and embeds the $p$-adic equivariant homotopy category in the $p$-adic homotopy category of $\mathcal{B}$. Therefore, the main theorems are immediate corollaries of the following theorem that we prove in Sect. 5.

\section{Theorem 3.6.}

(i) The functor $\mathrm{A}_{0}$ embeds the full subcategory of simply connected finite $\mathbb{Q}$ type objects in the rational homotopy category of $\mathcal{B}$ as a full subcategory of the homotopy category of $\mathcal{A}_{0}$.

(ii) The functor $\mathrm{A}_{p}$ embeds the full subcategory of the simply connected finite p-type objects in the p-adic homotopy category of $\mathcal{B}$ as a full subcategory of the homotopy category of $\mathcal{A}_{p}$. 
As a consequence of Theorems 1.1, 1.3, 2.4, and 3.6, we obtain an embedding of the equivariant rational homotopy category of $G$-simply connected $G$-finite type $G$-spaces as a full subcategory of the homotopy category of $\mathcal{A}_{0}$. To complete the picture we need an intrinsic characterization of the $\mathcal{D}$-CDGAs in this subcategory. For this, recall that a graded commutative algebra is said to be finite type if it is finitely generated as a module in each degree and simply connected if it is concentrated in non-negative degrees and zero in degree 1 . We say that it is spacelike if in degree zero it is a cartesian product of copies of $\mathbb{Q}$. In Sect. 6, we prove the following characterization theorem.

Theorem 3.7. Let $\underline{R}$ be an object of $\mathcal{A}_{0}$. Then $\underline{R}$ is isomorphic in the homotopy category of $\mathcal{A}_{0}$ to $\underline{\mathrm{A}}_{0} X$ for some $G$-simply connected $G$-finite $\mathbb{Q}$-type space $X$ if and only if:

(i) $H^{*} \underline{R}(G / K[H])$ is finite type, simply connected, and spacelike for all $G / K[H]$ in $\mathcal{D}$,

(ii) The natural map $H^{0} \underline{R}(G / K[H]) \otimes H^{2} \underline{P}_{0}(G / K[H]) \rightarrow H^{2} \underline{R}(G / K[H])$ is injective for all $G / K[H]$ in $\mathcal{D}$, and

(iii) The natural map

$$
\operatorname{Tor}_{\underline{P}_{0}(G / K[K])}\left(\underline{P}_{0}(G / K[H]), \underline{R}(G / K[K])\right) \rightarrow \underline{R}(G / K[H])
$$

is a quasi-isomorphism for all $G / K[H]$ in $\mathcal{D}$.

Condition (i) ensures that each $\underline{R}(G / K[H])$ is equivalent to the De Rham functor applied to a simply connected finite type space. Condition (ii) is needed because the Borel construction of a $(G / H)_{e}$-space can be simply connected even when the original space is not. In condition (iii), Tor denotes the differential torsion product; this condition is the algebraic analogue of the condition 2.5 for an object of $\mathcal{B}$ to be in $\operatorname{Im}_{0}^{\Delta}$.

In the $\overline{\mathbf{F}}_{p}$ context, the cohomology of an $E_{\infty}$ algebra has an operation called $P^{0}$; we say that a finite type graded commutative algebra with an operation $P^{0}$ is spacelike when it is generated as an $\overline{\mathbf{F}}_{p}$-module by fixedpoints of $P^{0}$. We have an analogous characterization theorem also proved in Sect. 6.

Theorem 3.8. Let $\underline{R}$ be an object of $\mathcal{A}_{p}$. Then $\underline{R}$ is isomorphic in the homotopy category of $\mathcal{A}_{p}$ to $\underline{\mathrm{A}}_{p} X$ for some $G$-simply connected $G$-finite p-type space $X$ if and only if:

(ii) $H^{*} \underline{R}(G / K[H])$ is finite type, simply connected, and spacelike for all $G / K[H]$ in $\mathcal{D}$,

(ii) The natural map $H^{0} \underline{R}(G / K[H]) \otimes H^{2} \underline{P}_{p}(G / K[H]) \rightarrow H^{2} \underline{R}(G / K[H])$ is injective for all $G / K[H]$ in $\mathcal{D}$, and 
(iii) The natural map

$$
\operatorname{Tor}_{\underline{P}_{p}(G / K[K])}\left(\underline{P}_{p}(G / K[H]), \underline{R}(G / K[K])\right) \rightarrow \underline{R}(G / K[H])
$$

is a quasi-isomorphism for all $G / K[H]$,

In condition (iii), Tor denotes the $E_{\infty}$ torsion product (see for example $[6,3.1])$.

\section{Simplified diagrams}

The last section described our algebraic models in general, but in the case when the group $G$ is connected, that is, when $G$ is a torus $\mathbf{T}^{n}$, we can simplify these models and use smaller diagrams. The idea is to take advantage of the fact that the map $B(G / H) \rightarrow B(G / K)$ is a rational equivalence when the index of $H$ in $K$ is finite and is a $p$-adic equivalence when the index of $H$ in $K$ is prime to $p$. When $G$ is the circle $\mathbf{T}=S^{1}$, we can specify in detail a choice for the cofibrant approximation $\underline{P}_{0}$ for the simplified diagrams in the rational context, and we recover the $\mathbb{T}$-systems of the second author's thesis [9].

Let $\mathcal{D}_{0}$ be the subcategory of $\mathcal{D}$ consisting of only those objects $G / K[H]$ with $H$ connected, and let $\mathcal{D}_{p}$ be the subcategory of $\mathcal{D}$ of objects $G / K[H]$ with $\pi_{0} H$ a $p$-group. These subcategories will form the shape of our simplified diagrams. The inclusion of these categories in $\mathcal{D}$ induces functors $J_{0}$ and $J_{p}$ from $\mathcal{D}$-spaces to $\mathcal{D}_{0}$-spaces and $\mathcal{D}_{p}$-spaces. We let $\mathcal{B}_{0}^{\prime}$ and $\mathcal{B}_{p}^{\prime}$ be the over categories $\mathcal{D U} / J_{0} \underline{B}$ and $\mathcal{D U} / J_{p} \underline{B}$. We therefore obtain functors $J_{0}: \mathcal{B} \rightarrow \mathcal{B}_{0}^{\prime}$ and $J_{p}: \mathcal{B} \rightarrow \mathcal{B}_{p}^{\prime}$.

To compare the simplified categories $\mathcal{B}_{0}^{\prime}$ and $\mathcal{B}_{p}^{\prime}$ to the original $\mathcal{B}$ we produce functors going the other direction. Since we are assuming that $G$ is connected, the mapping sets in $\mathcal{D}, \mathcal{D}_{0}$, and $\mathcal{D}_{p}$ are either empty or a single point. It follows that we can define a functor $K_{0}: \mathcal{D}_{0} \mathcal{U} \rightarrow \mathcal{D U}$ by

$$
K_{0} \underline{Z}(G / K[H])=\underline{Z}\left(G / K\left[H_{e}\right]\right),
$$

where $H_{e}$ is the identity component of $H$. Similarly we can define a functor $K_{p}: \mathcal{D}_{p} \mathcal{U} \rightarrow \mathcal{D U}$ by

$$
K_{p} \underline{Z}(G / K[H])=\underline{Z}\left(G / K\left[H_{p}\right]\right),
$$

where $H_{p}$ denotes the $p$-Sylow subgroup of $H$, the subgroup of $H$ consisting of those components that are in the $p$-Sylow subgroup of $\pi_{0} H$. Since $H_{e} \subseteq$ $H_{p} \subseteq H$, we have maps in $\mathcal{D}$

$$
G / K[H] \rightarrow G / K\left[H_{p}\right] \rightarrow G / K\left[H_{e}\right]
$$

and these induce natural transformations $K_{0} J_{0} \rightarrow$ Id and $K_{p} J_{p} \rightarrow$ Id. Using these maps on $\underline{B}$, we obtain functors $K_{0}: \mathcal{B}_{0}^{\prime} \rightarrow \mathcal{B}$ and $K_{p}: \mathcal{B}_{p}^{\prime} \rightarrow \mathcal{B}$. 
Proposition 4.1. The functor $K_{0}$ embeds the rational homotopy category of $\mathcal{B}_{0}^{\prime}$ as a full subcategory of the rational homotopy category of $\mathcal{B}$. The functor $K_{p}$ embeds the p-adic homotopy category of $\mathcal{B}_{p}^{\prime}$ as a full subcategory of the p-adic homotopy category of $\mathcal{B}$.

Proof. The functors $J_{0}, J_{p}, K_{0}, K_{p}$ preserve the relevant equivalences and so induce functors on the relevant homotopy categories. The composite functors $J_{0} K_{0}$ and $J_{p} K_{p}$ are the identity functors, and the natural transformations $K_{0} J_{0} \rightarrow \mathrm{Id}$ and $K_{p} J_{p} \rightarrow \mathrm{Id}$ are isomorphisms on objects in the image of $K_{0}$ and $K_{p}$ respectively.

In order to understand the restriction of the functors $J_{0}$ and $J_{p}$ to $\operatorname{Im}_{0}^{\mathcal{B}}$ and $\operatorname{Im}_{p}^{\mathcal{B}}$, we need the following key fact that served as our motivation above.

Proposition 4.2. The map $K_{0} J_{0} \underline{B} \rightarrow \underline{B}$ (resp. $K_{p} J_{p} \underline{B} \rightarrow \underline{B}$ ) is a rational (resp. p-adic) equivalence.

Proof. The map

$$
B\left(G / H_{e}\right)=\underline{B}\left(G / K\left[H_{e}\right]\right) \rightarrow \underline{B}(G / K[H])=B(G / H)
$$

is an $H / H_{e}$-bundle. Since $H / H_{e}$ is a finite group that acts trivially on the homology of $B\left(G / H_{e}\right)$, this map is a rational equivalence. Similarly, $H / H_{p}$ is a finite group with order prime to $p$ that acts trivially on the homology of $B\left(G / H_{p}\right)$, and so the map $\underline{B}\left(G / K\left[H_{p}\right]\right) \rightarrow \underline{B}(G / K[H])$ is a $p$-adic equivalence.

We define $\operatorname{Im}_{0}^{\mathcal{B}_{0}^{\prime}}$ to be the full subcategory of the rational homotopy category of $\mathcal{B}_{0}^{\prime}$ consisting of those objects $\underline{Z}$ for which the maps

$$
\underline{Z}(G / K[H]) \rightarrow \underline{B}(G / K[H]) \times_{\underline{B}}(G / K[K]) \underline{Z}(G / K[K]) .
$$

are rational equivalences for all $G / K[H]$ in $\mathcal{D}_{0}$. Likewise, define $\operatorname{Im}_{p}^{\mathcal{B}_{p}^{\prime}}$ to be the full subcategory of the $p$-adic homotopy category of $\mathcal{B}_{p}^{\prime}$ consisting of those objects for which the analogous maps are $p$-adic equivalences for all $G / K[H]$ in $\mathcal{D}_{p}$. Then the previous proposition and the Eilenberg-Moore spectral sequence give the following.

Proposition 4.3. The functors $J_{0}$ and $K_{0}$ restrict to inverse equivalences of $\operatorname{Im}_{0}^{\mathcal{B}_{0}^{\prime}}$ and $I m_{0}^{\mathcal{B}}$. The functors $J_{p}$ and $K_{p}$ restrict to inverse equivalences of $\operatorname{Im}_{p}^{\mathcal{B}_{p}^{\prime}}$ and $\operatorname{Im}_{p}^{\mathcal{B}}$.

In algebra, we have analogous functors $J_{*}$ and $K_{*}$ between the functor categories on $\mathcal{D}$ and the functor categories on $\mathcal{D}_{0}$ and $\mathcal{D}_{p}$. We abbreviate 
$J_{0} \underline{P}_{0}$ and $J_{p} \underline{P}_{p}$ to $\underline{P}_{0}^{\prime}$ and $\underline{P}_{p}^{\prime}$, and we let $\mathcal{A}_{0}^{\prime}$ and $\mathcal{A}_{p}^{\prime}$ denote the categories of $\mathcal{D}_{0}$-CDGAs under $\underline{P}_{0}^{\prime}$ and of $\mathcal{D}$ - $E_{\infty}$ DGAs under $\underline{P}_{p}^{\prime}$ respectively. The same argument as that of Proposition 4.1 shows that $K_{0}$ and $K_{p}$ embed the homotopy categories of $\mathcal{A}_{0}^{\prime}$ and $\mathcal{A}_{p}^{\prime}$ in the homotopy categories of $\mathcal{A}_{0}$ and $\mathcal{A}_{p}$ respectively. We obtain the following version of our main theorems.

Theorem 4.4. Let $G$ be a torus.

(i) The functor $\underline{\mathrm{A}}_{0}$ from the equivariant rational homotopy category to the homotopy category of $\mathcal{A}_{0}^{\prime}$ is full and faithful on the full subcategory of $G$-simply connected $G$-finite $\mathbb{Q}$-type $G$-spaces.

(ii) The functor $\underline{A}_{p}$ from the equivariant $p$-adic homotopy category to the homotopy category of $\mathcal{A}_{p}^{\prime}$ is full and faithful on the full subcategory of $G$-simply connected $G$-finite p-type $G$-spaces.

The obvious analogues of the characterization Theorems 3.7 and 3.8 are also immediate consequences.

Finally, we close this section with a concrete description of the rational models we obtain when $G$ is the circle group $\mathbb{T}=S^{1}$. Since $\mathbb{T}$ has precisely two connected subgroups, $e$ and $\mathbb{T}$, the category $\mathcal{D}_{0}$ therefore consists of the objects $G / H[e]$ for $H \subseteq \mathbb{T}$ and the object $G / \mathbb{T}[\mathbb{T}]$. Observe that $J_{0} \underline{B}(G / H[e])=B \mathbb{T}$ and the map induced by $G / K[e] \rightarrow G / H[e]$ is the identity. $J_{0} \underline{B}(G / \mathbb{T}[\mathbb{T}])=B(\mathbb{T} / \mathbb{T})=*$. Since $H^{*} B \mathbb{T}$ is the polynomial algebra on a generator $c$ in degree 2, choosing a representing cycle gives a map of CDGA's from $\mathbb{Q}[c]$ to $\Omega^{*} B \mathbb{T}$. The unit map $\mathbb{Q} \rightarrow \Omega^{*} *$ is an isomorphism.

Definition 4.5 (Rational models for the circle group). Define the $\mathcal{D}_{0}$ $C D G A \underline{P}_{0}^{\prime}$ by $\underline{P}_{0}^{\prime}(G / H[e])=\mathbb{Q}[c]$, and $\underline{P}_{0}^{\prime}(G / \mathbb{T}[\mathbb{T}])=\mathbb{Q}$. This is a cofibrant approximation of $J_{0} \underline{\Omega}^{*} \underline{B}$. The category $\mathcal{A}_{0}^{\prime}$ is the under category of $\mathcal{D}_{0}$-CDGAs, under this $\underline{P}_{0}^{\prime}$.

This is the category described in [9].

\section{Proof of Theorem 3.6}

Theorem 3.6 compares an over category of diagrams of spaces with under categories of diagrams of algebras. In the present context where our objects are indexed on the discrete diagram $\mathcal{D}$, we can use the singular complex and geometric realization functors to translate the problem into the analogous problem for simplicial sets. Let $\mathfrak{S}$ denote the category of simplicial sets, let $\mathcal{D S}$ denote the category of $\mathcal{D}$ simplicial sets, the category of contravariant functors from $\mathcal{D}$ to $\mathfrak{S}$. We denote by $\underline{B}_{\mathrm{S}}$ the singular complex of $\underline{B}$; in other words, $\underline{B}_{\mathrm{S}}$ is the $\mathcal{D}$ simplicial set with $\underline{B}_{\mathrm{S}}(G / K[H])$ the singular complex of $\underline{B}(G / K[H])$. Let $\mathcal{B}_{\mathrm{s}}$ be the category $\mathcal{D} \mathfrak{S} / \underline{B}_{\mathrm{s}}$ of $\mathcal{D}$ simplicial sets lying 
over $\underline{B}_{\mathrm{s}}$. We can regard the singular complex as a functor from $\mathcal{B}$ to $\mathcal{B}_{\mathrm{s}}$; as such, it preserves weak equivalences, rational equivalences, and $p$-adic equivalences. Standard arguments give the following observation.

Proposition 5.1. The singular complex functor induces equivalences of:

(i) The homotopy category of $\mathcal{B}$ and the homotopy category of $\mathcal{B}_{\mathrm{s}}$.

(ii) The rational homotopy category of $\mathcal{B}$ and the rational homotopy category of $\mathcal{B}_{\mathrm{s}}$.

(iii) The p-adic homotopy category of $\mathcal{B}$ and the p-adic homotopy category of $\mathcal{B}_{\mathrm{s}}$.

The functors $A_{0}$ and $A_{p}$ that we consider in Theorem 3.6 are defined by objectwise application of a functor on simplicial sets to the singular complex of an object of $\mathcal{B}$. In other words, both of these functors factor through the category $\mathcal{B}_{\mathrm{s}}$. We denote the corresponding functors on $\mathcal{B}_{\mathrm{s}}$ by the same symbols: $\mathrm{A}_{0}$ applies the polynomial De Rham functor $\Omega^{*}$ and $\mathrm{A}_{p}$ applies the cochain functor $C^{*}$ objectwise to an object of $\mathcal{B}$. Thus, to prove Theorem 3.6, it suffices to prove the following simplicial analogue.

\section{Theorem 5.2.}

(i) The functor $\mathrm{A}_{0}$ embeds the full subcategory of simply connected finite $\mathbb{Q}$-type objects in the rational homotopy category of $\mathcal{B}_{\mathrm{s}}$ as a full subcategory of the homotopy category of $\mathcal{A}_{0}$.

(ii) The functor $\mathrm{A}_{p}$ embeds the full subcategory of the simply connected finite p-type objects in the p-adic homotopy category of $\mathcal{B}_{\mathrm{s}}$ as a full subcategory of the homotopy category of $\mathcal{A}_{p}$.

The advantage of working in the simplicial context is that now the functors $\mathrm{A}_{0}$ and $\mathrm{A}_{p}$ have adjoints. In [1], Bousfield and Gugenheim construct an adjoint $U_{0}$ to the De Rham functor and essentially the same construction in [6] gives an adjoint $U_{p}$ to the cochain functor. Let $\underline{U}_{0}$ and $\underline{U}_{p}$ denote the functors from $\mathcal{D}$-CDGAs and $\mathcal{D}$ - $E_{\infty}$ DGAs to $\mathcal{D} \mathfrak{S}$ obtained by applying $U_{0}$ and $U_{p}$ objectwise. An easy exercise in category theory proves that these functors are then adjoint to the functors $\underline{\Omega}^{*}$ and $\underline{C}^{*}$ defined in 3.2. Since $\underline{U}_{0} \underline{P}_{0}$ is generally not $\underline{B}_{\mathrm{s}}, \underline{U}_{0}$ does not define a functor from $\mathcal{A}_{0}$ to $\mathcal{B}_{\mathrm{s}}$. On the other hand, the $\left(\underline{\Omega}^{*}, \underline{U}_{0}\right)$ adjunction does give us a map $\underline{B}_{\mathrm{S}} \rightarrow \underline{U}_{0} \underline{\Omega}^{*} \underline{B}_{\mathrm{S}} \rightarrow \underline{U}_{0} \underline{P}_{0}$, and so we can define a functor $V_{0}: \mathcal{A}_{0} \rightarrow \mathcal{B}_{\mathrm{s}}$ by

$$
V_{0} \underline{R}=\underline{B}_{\mathrm{S}} \times_{\underline{U}_{0} \underline{P}_{0}} \underline{U}_{0} \underline{R} .
$$

A check of universal properties shows that $V_{0}$ is adjoint to $A_{0}$. Similarly, we define $V_{p}: \mathcal{A}_{p} \rightarrow \mathcal{B}_{\mathrm{s}}$ by $V_{p} \underline{R}=\underline{B}_{\mathrm{S}} \times_{\underline{U}_{p}} \underline{P}_{p} \underline{U_{p}} \underline{R}$, and $V_{p}$ is adjoint to $\mathrm{A}_{p}$.

The next obstacle is that the functors $V_{0}$ and $V_{p}$ do not preserve weak equivalences. In fact, the functors $U_{0}$ and $U_{p}$ already do not preserve weak 
equivalences. This is handled in [10] by using minimal models, and similarly in [1] and [6] by using cofibrant approximation. It is shown in [1] and [6] that $U_{0}$ and $U_{p}$ do preserve weak equivalences between cofibrant objects. In current jargon, the $\left(\underline{\Omega}^{*}, \underline{U}\right)$ and $\left(\underline{C}^{*}, \underline{U}\right)$ adjunctions form Quillen adjoint pairs, which means that in addition $U_{0}$ and $U_{p}$ convert cofibrations to Kan fibrations of simplicial sets. We recall the definition of cofibration of CDGA's.

Definition 5.3. Let $A \rightarrow B$ be a map of CDGA's. Let $A^{\sharp}$ denote the underlying graded commutative algebra of $A$. We say that $A \rightarrow B$ is cellular if there exist graded submodules $\left(X_{0}=0\right), X_{1}, X_{2}, \ldots$ of the underlying graded module of $B$ such that

(i) The differential of any element in $X_{n}$ is in the sub-graded $A^{\sharp}$-algebra generated by $X_{0}, \ldots, X_{n-1}$.

(ii) The underlying graded $A^{\sharp}$ algebra of $R$ is the free graded commutative $A^{\sharp}$ algebra on $X_{1} \oplus X_{2} \oplus \cdots$.

We say that $A \rightarrow B$ is a cofibration (written $A \longmapsto B$ ) if it is the retract of some cellular map $A^{\prime} \rightarrow B^{\prime}$.

The definition of cofibration of $E_{\infty}$ algebras is entirely similar. The definition of cofibration is just a relative form of the definition of cofibrant: An object is cofibrant if and only if the map from the initial object is a cofibration. Although it may not be obvious from the definition given above, it turns out that the composition of cofibrations is a cofibration; this is one of the axioms of a closed model category structure.

A relative form of the construction of the cofibrant approximation functor alluded to in section 3 gives a factorization functor that takes a map $f: A \rightarrow$ $B$ to the composite of a cofibration $A \longmapsto B^{\prime}$ and a weak equivalence $B^{\prime} \rightarrow$ $B$, functorially in $f$. Functoriality here means that when the diagram on the left commutes, the construction gives a map $B^{\prime} \rightarrow D^{\prime}$ that makes the factorization diagram on the right commute.
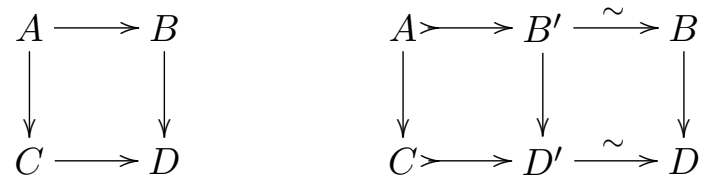

Applying this to $\mathcal{D}$ diagrams, we obtain objectwise cofibrant approximation functor in $\mathcal{A}_{0}$.

Proposition 5.4. There exists a functor $\underline{\Gamma}_{0}: \mathcal{A}_{0} \rightarrow \mathcal{A}_{0}$ and a natural quasi-isomorphism $\gamma: \underline{\Gamma}_{0} \rightarrow I d$, such that for every $\underline{R}$, the initial map $\underline{P}_{0} \rightarrow \underline{\Gamma}_{0} \underline{R}$ is an objectwise cofibration, that is, the map $\underline{P}_{0}(G / K[H]) \rightarrow$ $\left(\underline{\Gamma}_{0} \underline{R}\right)(G / K[H])$ is a cofibration of $C D G A$ 's for every $G / K[H]$ in $\mathcal{D}$. 
Similarly, we obtain an objectwise cofibrant approximation functor $\underline{\Gamma}_{p}$ in the category $\mathcal{A}_{p}$.

Proposition 5.5. The functor $V_{0} \underline{\Gamma}_{0}$ converts quasi-isomorphisms in $\mathcal{A}_{0}$ to rational equivalences in $\mathcal{B}_{\mathrm{s}}$. The functor $V_{p} \underline{\Gamma}_{p}$ converts quasi-isomorphisms in $\mathcal{A}_{p}$ to p-adic equivalences in $\mathcal{B}_{\mathrm{s}}$.

Proof. The functor $U_{0}$ preserves weak equivalences between cofibrant CDGA's and converts cofibrations of CDGA's to Kan fibrations. It follows that $\underline{U}_{0} \underline{\Gamma}_{0}$ preserves weak equivalences between objectwise cofibrant objects and converts objectwise weak equivalences to objectwise Kan fibrations. Thus, $\underline{U}_{0} \underline{P}_{p}(G / K[H])$ is a Kan complex and $\underline{U}_{0} \underline{\Gamma}_{0} \underline{R}(G / K[H]) \rightarrow$ $\underline{U}_{0} \underline{P}_{p}(G / K[H])$ is a Kan fibration for every $\underline{R}$ in $\mathcal{A}_{0}$ and every $G / K[H]$ in $\mathcal{D}$. Since $\underline{B}_{\mathrm{S}}$ is simply connected, $\underline{U}_{0} \underline{P}_{0}$ is simply connected, and it follows from the Eilenberg-Moore spectral sequence that the pullback $\underline{B}_{\mathrm{S}} \times_{\underline{U}_{0}} \underline{\underline{P}}_{0}(-)$ preserves rational equivalences. We conclude that

$$
V_{0} \underline{\Gamma}_{0}(-)=\underline{B}_{\mathrm{S}} \times_{\underline{U}_{0}} \underline{P}_{0} \underline{U}_{0} \underline{\Gamma}_{0}(-)
$$

converts quasi-isomorphisms to rational equivalences. The $p$-adic case is entirely similar.

We obtain an induced functor $\mathbf{V}_{0}$ from the homotopy category of $\mathcal{A}_{0}$ to the rational homotopy category of $\mathcal{B}_{\mathrm{s}}$, and an induced functor $\mathbf{V}_{p}$ from the homotopy category of $\mathcal{A}_{p}$ to the $p$-adic homotopy category of $\mathcal{B}_{\mathrm{s}}$. A standard model category argument then gives the following result, but we include a self-contained proof.

Proposition 5.6. The derived functors $\left(\mathrm{A}_{0}, \mathrm{~V}_{0}\right)$ and $\left(\mathrm{A}_{p}, \mathbf{V}_{p}\right)$ are adjoint pairs.

Proof. Let $\eta:$ Id $\rightarrow V_{0} \mathrm{~A}_{0}$ and $\epsilon: \mathrm{Id} \rightarrow \mathrm{A}_{0} V_{0}$ be the unit and counit of the $\left(\mathrm{A}_{0}, V_{0}\right)$ adjunction. We obtain a natural transformation $\epsilon^{\prime}: \underline{\Gamma}_{0} \rightarrow \mathrm{A}_{0} V_{0} \underline{\Gamma_{0}}$, and using the natural transformation $\gamma \underline{\Gamma}_{0} \rightarrow \mathrm{Id}$, we obtain a natural transformation $\eta^{\prime}:$ Id $\rightarrow V_{0} \underline{\Gamma}_{0} \mathrm{~A}_{0}$. For an object $\underline{Y}$ in $\mathcal{B}_{\mathrm{s}}$, the composite

$$
\underline{\Gamma}_{0} \mathrm{~A}_{0} \underline{Y} \stackrel{\epsilon^{\prime}}{\rightarrow} \mathrm{A}_{0} V_{0} \underline{\Gamma}_{0} \mathrm{~A}_{0} \stackrel{\mathrm{A}_{0} \eta^{\prime}}{\rightarrow} \mathrm{A}_{0} \underline{Y}
$$

is the natural transformation $\gamma$. Conversely, for an object $\underline{R}$ in $\mathcal{A}_{0}$, the composite

$$
V_{0} \underline{\Gamma}_{0} \underline{R} \stackrel{\eta^{\prime}}{\rightarrow} V_{0} \underline{\Gamma}_{0} \mathrm{~A}_{0} V_{0} \underline{\Gamma}_{0} \underline{R} \stackrel{V_{0} \underline{\Gamma}_{0} \epsilon^{\prime} \gamma}{\longrightarrow} V_{0} \underline{\Gamma}_{0} \underline{R}
$$

is the identity. In the homotopy category of $\mathcal{A}_{0}$, the natural map $\gamma$ is an isomorphism, and using the inverse natural isomorphism $\gamma^{-1}$, an easy check verifies that the derived functors $A_{0}$ and $\mathbf{V}_{0}$ are adjoint. The argument in the $p$-adic case is entirely similar. 
We can now prove Theorem 5.2.

Proof (Proof of Theorem 5.2). We treat the rational case in detail; the $p$-adic case follows by analogous arguments. Using the previous proposition, it suffices to show that the unit of the adjunction

$$
\eta^{\prime}: \underline{Y} \rightarrow V_{0} \underline{\Gamma}_{0} \mathrm{~A}_{0} \underline{Y}
$$

is a rational equivalence whenever $\underline{Y}$ is a simply connected and finite $\mathbb{Q}$-type object of $\mathcal{B}_{\text {s. }}$.

By construction, each CDGA $\left(\underline{\Gamma}_{0} \mathrm{~A}_{0} \underline{Y}\right)(G / K[H])$ is a cofibrant approximation of $\left(\mathrm{A}_{0} \underline{Y}\right)(G / K[H])=\Omega^{*}(\underline{Y}(G / K[H]))$. The main argument of [1] is that the map

$$
\underline{Y}(G / K[H]) \rightarrow U_{0}\left(\underline{\Gamma}_{0} \mathrm{~A}_{0} \underline{Y}\right)(G / K[H])
$$

is a rational equivalence since $\underline{Y}(G / K[H])$ is a simply connected finite $\mathbb{Q}$-type space. Since $U_{0}$ converts cofibrations to Kan fibrations, the map

$$
\underline{U}_{0}\left(\underline{\Gamma}_{0} \mathrm{~A}_{0} \underline{Y}\right)(G / K[H]) \rightarrow \underline{U}_{0} \underline{P}_{0}
$$

is a Kan fibration between simply connected Kan complexes, and it follows that the maps

$\underline{Y}(G / K[H]) \rightarrow \underline{B}_{\mathrm{S}} \times_{\underline{U}_{0}} \underline{\underline{P}}_{0} \underline{U}_{0} \underline{\Gamma}_{0} \mathrm{~A}_{0} \underline{Y}(G / K[H]) \rightarrow U_{0} \underline{\Gamma}_{0} \mathrm{~A}_{0} \underline{Y}(G / K[H])$

are rational equivalences. Since the first map is $\eta^{\prime}$ on $G / K[H]$, it follows that $\eta^{\prime}$ is a rational equivalence.

\section{The sections functor and the proof of the characterization theorems}

In this section, we prove the characterization Theorems 3.7 and 3.8. It turns out that it is much easier to prove these theorems using the category of $\Delta \mathcal{O}_{G^{-}}$ spaces rather than the category $\mathcal{B}$. For this reason, we introduce the sections functor $S$ inverse to the bundle functor $Q$. This functor is also exactly what is needed to prove Theorems 2.4 and 2.5 from Sect. 2, giving the passage from diagrams of spaces to bundles, and so we begin with the proof of these theorems.

The most concise way to define the functor $S$ is to note that the map that takes a space to its set of components defines a functor $\Delta \mathcal{O}_{G} \rightarrow \mathcal{D}$ that allows us to regard a $\mathcal{D}$-space as a $\Delta \mathcal{O}_{G}$-space. Recall that $\underline{E}$ is the $\Delta \mathcal{O}_{G}$-space defined in (2.1) as $\underline{E}(G / K[H])=E(G / H)$, and $\underline{B}$ is given by $\underline{B}(G / K[H])=E(G / H) /(G / H)_{e}$ (as a $\pi_{0} G$ space). Then we have a map of $\Delta \mathcal{O}_{G}$-spaces $\underline{E} \rightarrow \underline{B}$. For an object $\underline{Y}$ of $\mathcal{B}$, we define

$$
S \underline{Y}=\underline{E} \times \underline{B} \underline{Y},
$$


the pullback in the category of $\Delta \mathcal{O}_{G}$-spaces. In concrete terms

$$
\begin{aligned}
S \underline{Y}(G / K[H]) & =\underline{E}(G / K[H]) \times_{\underline{B}(G / K[H])} \underline{Y}(G / K[H]) \\
& =\underline{E}(G / H) \times_{\left(E(G / H) /(G / H)_{e}\right)} \underline{Y}(G / K[H]) .
\end{aligned}
$$

Then $S$ defines a functor from $\mathcal{B}$ to $\Delta \mathcal{O}_{G} \mathcal{U}$.

The map $E(G / H) \rightarrow \underline{E}(G / H) /(G / H)_{e}$ is a fibration, and so the functor $S$ preserves weak equivalences. Moreover, $E(G / H) /(G / H)_{e}$ is a model for $B(G / H)_{e}$ and is therefore connected and simply connected; it follows from the Eilenberg-Moore spectral sequence that $S$ also preserves rational equivalences and $p$-adic equivalences.

Proof (Proof of 2.4). Consider the composite functor $S Q$. For any $\Delta \mathcal{O}_{G^{-}}$ space $\underline{X}$, we have

$$
\begin{aligned}
& S Q \underline{X}(G / K[H]) \\
& \begin{array}{r}
\underline{E}(G / K[H]) \times_{\left(\underline{E}(G / K[H]) \times(G / H) e^{*}\right)}\left(\underline{E}(G / K[H]) \times(G / H)_{e} \underline{X}(G / K[H])\right) \\
\cong \underline{E}(G / K[H]) \times \underline{X}(G / K[H]) \\
=(\underline{E} \times \underline{X})(G / K[H]) .
\end{array}
\end{aligned}
$$

These isomorphisms are natural in $G / K[H]$ and so we obtain a natural isomorphism $S Q \underline{X} \cong \underline{E} \times \underline{X}$. The weak equivalence $\underline{E} \rightarrow \underline{*}$ induces a natural weak equivalence $S Q \rightarrow \mathrm{Id}$.

Now consider the other composite. By definition,

$$
\begin{aligned}
Q S Y(G / K[H]) & =E(G / H) \times_{(G / H)_{e}} S Y(G / K[H]) \\
& \cong E(G / H) \times_{(G / H)_{e}}\left(E(G / H) \times_{\underline{B}(G / K[H])} Y(G / K[H])\right) \\
& \cong\left(E(G / H) \times_{(G / H)_{e}} E(G / H)\right) \times_{\underline{B}(G / K[H])} Y(G / K[H])
\end{aligned}
$$

There is a homotopy equivalence

$$
\underline{B}(G / K[H])=E(G / H) /(G / H)_{e} \rightarrow E(G / H) \times_{(G / H)_{e}} E(G / H)
$$

whose composite with both projection maps to $\underline{B}(G / K[H])$ is the identity; it is constructed using the diagonal map of $E(G / H)$. Using this, we can define a map $Y(G / K[H]) \rightarrow Q S Y(G / K[H])$ which commutes with the projections to $\underline{B}(G / K[H])$, and this map is also a homotopy equivalence. These maps fit together to give a natural weak equivalence Id $\rightarrow Q S$.

Proof. (Proof of 2.6.) An easy bundle argument shows that the functor $Q$ takes objects in $\operatorname{Im}^{\Delta}, \operatorname{Im}_{0}^{\Delta}$, and $\operatorname{Im}_{p}^{\Delta}$ to objects in $\operatorname{Im}^{\mathcal{B}}, \operatorname{Im}_{0}^{\mathcal{B}}$, and $\operatorname{Im}_{p}^{\mathcal{B}}$. Likewise, an easy bundle argument together with the Eilenberg-Moore spectral sequence implies that the functor $S$ takes objects in $\operatorname{Im}^{\mathcal{B}}, \operatorname{Im}_{0}^{\mathcal{B}}$, and $\operatorname{Im}{ }_{p}^{\mathcal{B}}$ to objects in $I m^{\Delta}, I m_{0}^{\Delta}$, and $I m_{p}^{\Delta}$. 
Now we move on to the proof of the characterization theorems.

Proof (Proof of Theorems 3.7 and 3.8).

Let $X$ be a $G$-simply connected and $G$-finite $\mathbb{Q}$ - or $p$ - type $G$-space. Then $H^{*} \underline{\mathrm{A}}_{0} X$ and $H^{*} \underline{\mathrm{A}}_{p} X$ are isomorphic as commutative algebras to the cohomology of Borel constructions on $X^{K}$; since these are simply connected and finite $\mathbb{Q}$ - or $p$ - type spaces, their cohomology satisfies condition (i). We have that each space $X^{K}$ is (non-equivariantly) homotopy equivalent to the fiber of the map $I \Phi X(G / K[H]) \rightarrow \underline{B}(G / K[H])$, and so its $\mathbb{Q}$ - or $\mathbb{Z} / p \mathbb{Z}$-cohomology is calculated by the Eilenberg-Moore spectral sequence. Looking at this spectral sequence, we see that $H^{1} X^{K}$ is the kernel of the differential

$$
H^{0} X^{K} \otimes H^{2} \underline{B}(G / K[H]) \rightarrow H^{2} X^{K} .
$$

Since each $X^{K}$ is simply connected, condition (ii) holds. Finally, we have that $I \Phi X(G / K[H])$ is isomorphic to the fiber product

$$
\underline{B}(G / K[H]) \times_{\underline{B}(G / K[K])} I \Phi X(G / K[K])
$$

and the map $I \Phi X(G / K[K]) \rightarrow \underline{B}(G / K[K])$ is a fibration, and so by [1, $3.1]$ or $[6,5.2]$, condition (iii) holds.

In proving the converse, consider first the rational case. Suppose $\underline{R}$ satisfies conditions (i), (ii), and (iii). We can assume without loss of generality that $\underline{R}$ is objectwise cofibrant by replacing $\underline{R}$ by $\underline{\Gamma}_{0} \underline{R}$ if necessary. Now $\underline{B}$ is not rational, but $\underline{E}$ is, and so (as mentioned above) it is much easier to make the arguments in the category of $\Delta \mathcal{O}_{G}$-spaces than it is in $\mathcal{B}$. Let $\underline{X}=S\left|V_{0} \underline{R}\right|$ where $|\cdot|$ denotes geometric realization. Explicitly,

$$
\underline{X}=\underline{E} \times{ }_{\underline{B}}\left|\underline{B}_{\mathrm{S}} \times_{\underline{U}_{0} \underline{P}_{0}} \underline{U}_{0} \underline{R}\right| \cong\left(\underline{E} \times \underline{B}_{\underline{B}}\left|\underline{B}_{\mathrm{S}}\right|\right) \times_{\left|\underline{U}_{0} \underline{P}_{0}\right|}\left|\underline{U}_{0} \underline{R}\right| .
$$

It is convenient to abbreviate $\underline{E} \times{ }_{\underline{B}}\left|\underline{B}_{\mathrm{S}}\right|$ to $\underline{E^{\prime}}$. Since $\underline{E} \rightarrow \underline{B}$ is an objectwise fibration and $\left|\underline{B}_{\mathrm{S}}\right| \rightarrow \underline{B}$ is an objectwise weak equivalence, we have that $\underline{E}^{\prime}$ is objectwise contractible. It follows that $\underline{X}(G / K[H])$ is a rational space for all $G / K[H]$. By condition (i), $\underline{U}_{0} \underline{R}(G / K[H])$ is finite $\mathbb{Q}$-type and simply connected for all $G / K[H]$, and so $\underline{X}$ is finite $\mathbb{Q}$-type. To see that $\underline{X}$ is simply connected, it suffices to show that $H^{1} X(G / K[H])=0$, and this follows from condition (ii) and the Eilenberg-Moore spectral sequence (and $[1,3.1])$. Finally, condition (iii) implies that the map

$$
\underline{U}_{0} \underline{R}(G / K[H]) \rightarrow \underline{U}_{0} \underline{P}_{0}(G / K[H]) \times_{\underline{U}_{0} \underline{P}_{0}(G / K[K])} \underline{U}_{0} \underline{R}(G / K[K])
$$

is a rational equivalence, and so the map

$$
\begin{aligned}
& \underline{X}(G / K[H]) \rightarrow\left(\underline{E}^{\prime}(G / K[H]) \times_{\underline{U}_{0} \underline{P}_{0}(G / K[K])} \underline{U}_{0} \underline{R}(G / K[K])\right. \\
& \rightarrow \underline{E}^{\prime}(G / K[K]) \times_{\underline{U}_{0} \underline{P}_{0}(G / K[K])} \underline{U}_{0} \underline{R}(G / K[K])=\underline{X}(G / K[K]),
\end{aligned}
$$


obtained by pulling back along the map $\underline{E}^{\prime}(G / K[H]) \rightarrow \underline{P}_{0}(G / K[H])$, is a rational equivalence. Thus, $X$ is a finite $\mathbb{Q}$-type simply connected object of $I m_{0}^{\Delta}$. Therefore $\underline{X}$ is rationally equivalent to $I \Phi X$ for some $G$-simply connected $G$-finite $\mathbb{Q}$-type $G$-space $X$. We have quasi-isomorphisms in $\mathcal{A}_{0}$

$$
\underline{R} \stackrel{\sim}{\rightarrow} \mathrm{A}_{0} V_{0} \underline{R} \stackrel{\sim}{\rightarrow} \mathrm{A}_{0} Q S\left|V_{0} \underline{R}\right|=\mathrm{A}_{0} Q \underline{X} \simeq \mathrm{A}_{0} Q I \Phi X=\underline{\mathrm{A}}_{0} X .
$$

The $p$-adic case is identical, with $[6,5.2]$ taking the place of $[1,3.1]$.

\section{Proof of Theorems 1.3 and 1.4}

In this section, we prove the equivalences of categories claimed in Sect. 1. We prove both equivalences together, by constructing a left inverse for $I$ on the various homotopy categories.

The first step is to construct a functor $L$ from $\Delta \mathcal{O}_{G}$-spaces to $\mathcal{O}_{G}$-spaces and a natural transformation $L I \rightarrow$ Id that is always a weak equivalence. We construct $L$ as a homotopy colimit. $L$ preserves weak equivalences, rational equivalences, and $p$-adic equivalences, and so it induces a functor on the various homotopy categories. This gives us retractions. To prove the equivalences, we need to analyze the other composite $I L$. Rather than comparing $I L$ to the identity functor directly, we must construct an intermediate functor $\Psi$ from $\Delta \mathcal{O}_{G} \mathcal{U}$ to itself and natural transformations

$$
\text { Id } \stackrel{\psi}{\leftarrow} \Psi \stackrel{\alpha}{\rightarrow} I L
$$

We show that the backwards transformation $\psi$ is homotopy equivalence at each object. It follows that the functor $\Psi$ preserves weak equivalences, rational equivalences, and $p$-adic equivalences, and so induces a functor on the various homotopy categories. The zigzag (7.1) then defines a natural transformation $\eta$ : Id $\rightarrow I L$ in each of these homotopy categories. Finally we show that $\alpha$ is a weak equivalence, rational equivalence, and $p$-adic equivalence at each object when we restrict to the subcategories $\operatorname{Im}^{\Delta}, I m_{0}^{\Delta}$, and $\operatorname{Im}_{p}^{\Delta}$ respectively. We conclude that $\eta$ is an isomorphism exactly for the objects in $I m^{\Delta}, I m_{0}^{\Delta}$, and $I m_{p}^{\Delta}$ respectively (as it cannot be an isomorphism for objects not in $\operatorname{Im}^{\Delta}, \operatorname{Im}_{0}^{\Delta}$, and $\operatorname{Im}_{p}^{\Delta}$ ). Theorems 1.3 and 1.4 are immediate consequences.

Since our arguments make extensive use of homotopy colimits, we begin by recalling a few facts about them. First, since we are working with contravariant functors to spaces, we have:

Lemma 7.2. If $C$ is a category with initial object $c \in C$ and $X$ is a contravariant functor from $C$ to spaces, then the inclusion $X(c) \rightarrow \operatorname{Hocolim}_{C} X$ is a homotopy equivalence. 
We understand Hocolim always to denote the geometric realization of the usual categorical bar construction. We will use the following observation extensively to construct maps between homotopy colimits in what follows.

Observation. Let $C$ and $D$ be categories, and $X$ and $Y$ contravariant functors from $C$ and $D$ to spaces respectively. If $F$ is a functor $F: C \rightarrow D$, we denote the composite functor $Y \circ F$ from $D$ to spaces as $F^{*} Y$. Then a natural transformation from $X$ to $F^{*} Y$ induces a map $\operatorname{Hocolim}_{C} X \rightarrow$ $\operatorname{Hocolim}_{D} Y$.

We use the notion of a left cofinal functor introduced by Bousfield-Kan in [2]. Given a functor $F: A \rightarrow B$ and an object $b \in B$, let $F \downarrow b$ denote the category whose objects are pairs $(a, \phi)$ where $a \in A$ and $\phi: F(a) \rightarrow b$ is a map in $B$. Morphisms in $F \downarrow b$ between $\left(a_{1}, \phi_{1}\right)$ and $\left(a_{2}, \phi_{2}\right)$ are given by maps $\alpha: a_{1} \rightarrow a_{2}$ in $A$ such that $\phi_{2} F(\alpha)=\phi_{1}$. The functor $F$ is left cofinal if for every object $b \in B$, the nerve of the category $F \downarrow b$ is contractible. This notion is useful because of the following:

Lemma 7.3. If $F: A \rightarrow B$ is a left cofinal functor and $X$ is a contravariant functor from $B$ to spaces, then the induced map

$$
\operatorname{Hocolim}_{A} F^{*} X \rightarrow \operatorname{Hocolim}_{B} X
$$

is a weak equivalence.

Proof. The proof follows that of Theorem XI.9.2 in [2]. The only difference is that we are dealing with contravariant functors and direct limits rather than covariant functors and inverse limits.

We now describe the construction of $L$. Let $\mathcal{E}$ be the category which has the same objects as $\mathcal{O}_{G}$ but only the maps corresponding to the unit $e$ of $G$. Therefore $\mathcal{E}(G / H, G / K)$ is either a single map if $H \subseteq K$, or is empty. We define $\Delta \mathcal{E}$ similarly, as the category with objects the same as $\Delta \mathcal{O}_{G}$ and only the unit maps. Let $\mathcal{E}_{H}$ be the full subcategory of $\mathcal{E}$ with objects $G / A$ such that $H \subseteq A$; let $\Delta \mathcal{E}_{H}$ be the full subcategory of $\Delta \mathcal{E}$ with objects $G / B[A]$ such that $H \subseteq A$. Define $L \underline{X}(G / H)=\operatorname{Hocolim}_{\Delta \mathcal{E}_{H}} \underline{X}$.

To make $L$ into a functor on $\mathcal{O}_{G}$, observe that if $g: G / H \rightarrow G / K$ is a map in $\mathcal{O}_{G}$, then $H \subseteq K$ and $\Delta \mathcal{E}_{K}$ is a subcategory of $\Delta \mathcal{E}_{H}$. Moreover, the action of $g$ is well-defined on $\underline{X}(G / B[A])$ for $H \subseteq K \subseteq A \subseteq B$, and so $g$ induces a natural transformation on the restrictions $\underline{X}\left|\Delta \mathcal{E}_{K} \rightarrow \underline{X}\right|$ $\Delta \mathcal{E}_{H}$. Using the observation, we get induced structure maps $L \underline{X}(G / K) \rightarrow$ $L \underline{X}(G / H)$ from the inclusion of categories and the twisting action of the natural transformation. This makes $L \underline{X}$ into a functor on $\mathcal{O}_{G}$. Therefore $L$ defines a functor from $\Delta \mathcal{O}_{G} \mathcal{U}$ to $\mathcal{O}_{G} \mathcal{U}$.

Next we examine the composite functor $L I$. 
Proposition 7.4. There is a natural transformation $L I \rightarrow \mathrm{Id}$ that is a weak equivalence at each object.

Proof. The functor $I$ is defined as composition with the projection $\Delta \mathcal{O}_{G} \rightarrow$ $\mathcal{O}_{G}$. This projection also induces a projection $\Delta \mathcal{E}_{H} \rightarrow \mathcal{E}_{H}$. Therefore we have a natural map $\operatorname{Hocolim}_{\Delta \mathcal{E}_{H}} I \underline{X} \rightarrow \operatorname{Hocolim}_{\mathcal{E}_{H}} \underline{X}$. Observe that for any object $G / K \in \mathcal{E}_{H}$, the category $I \downarrow G / K$ has a final object given by $(G / K[H]$, id). Therefore the nerve of this category is contractible and so $\Delta \mathcal{E}_{H} \rightarrow \mathcal{E}_{H}$ is left cofinal. Invoking Lemma 7.3, we see that $\operatorname{Hocolim}_{\Delta \mathcal{E}_{H}} I \underline{X} \rightarrow$ Hocolim $_{\mathcal{E}_{H}} \underline{X}$ is a weak equivalence.

Since $G / H$ is the initial object in $\mathcal{E}_{H}$, the map $\operatorname{Hocolim}_{\mathcal{E}_{H}} \underline{X} \rightarrow \underline{X}(G / H)$ is a homotopy equivalence. The composite maps

$$
I \underline{X}(G / H)=\operatorname{Hocolim}_{\Delta \mathcal{E}_{H}} I \underline{X} \rightarrow \operatorname{Hocolim}_{\mathcal{E}_{H}} \underline{X} \rightarrow \underline{X}(G / H)
$$

fit together to give a natural transformation $L I \rightarrow$ Id which is a weak equivalence at each object.

The construction of the endofunctor $\Psi$ has a similar flavor. Recall that $\Delta \mathcal{E}$ denotes the subcategory of maps $e$ in $\Delta \mathcal{O}_{G}$, and define $\Delta \mathcal{E}_{G / K[H]}$ to be the full subcategory of $\Delta \mathcal{E}$ consisting of objects $G / B[H]$ with $B>K$. Define

$$
\Psi \underline{X}(G / K[H])=\operatorname{Hocolim}_{\Delta \mathcal{E}_{G / K[H]} \underline{X}}
$$

We make this a functor of $\Delta \mathcal{O}_{G}$ in the same way we did for $L$ : Given a map $g: G / K_{1}\left[H_{1}\right] \rightarrow G / K_{2}\left[H_{2}\right]$, we have a functor $\Delta \mathcal{E}_{G / K_{2}\left[H_{2}\right]} \rightarrow$ $\Delta \mathcal{E}_{G / K_{1}\left[H_{1}\right]}$ that takes the object $G / B\left[H_{2}\right]$ in $\Delta \mathcal{E}_{G / K_{2}\left[H_{2}\right]}$ to the object $G / B\left[H_{1}\right] \in \Delta \mathcal{E}_{G / K_{1}\left[H_{1}\right]}$; note that $B>K_{1}$ because $B>K_{2}>K_{1}$. Also, $g$ induces a twisting natural transformation via the action $\underline{X}\left(G / B\left[H_{2}\right]\right) \stackrel{g}{\rightarrow}$ $\underline{X}\left(G / B\left[H_{1}\right]\right)$. The $\Delta \mathcal{O}_{G}$ structure maps are induced by these as in the observation. This makes $\Psi$ into a functor from $\Delta \mathcal{O}_{G} \mathcal{U}$ to itself.

The category $\Delta \mathcal{E}_{G / K[H]}$ has an initial object $G / K[H]$. For any $G / B[H]$ in $\Delta \mathcal{E}_{G / K[H]}$, the initial map $G / K[H] \rightarrow G / B[H]$ induces a map $\underline{X}(G / B[H]) \rightarrow \underline{X}(G / K[H])$, and we obtain a map

$$
\Psi \underline{X}(G / K[H])=\operatorname{Hocolim}_{\Delta \mathcal{E}_{G / K[H]}} \underline{X} \rightarrow \underline{X}(G / K[H])
$$

that is a homotopy equivalence by Lemma 7.2. These maps assemble into the natural transformation $\psi: \Psi \rightarrow \mathrm{Id}$.

We define the natural transformation $\alpha$ as follows. For each $G>K>H$, consider the functor from $\Delta \mathcal{E}_{G / K[H]}$ to $\Delta \mathcal{E}_{K}$ that sends the object $G / B[H]$ to $G / B[K]$. Since $H \subseteq K \subseteq B$, we have a map $e: G / B[K] \rightarrow G / B[H]$ in $\Delta \mathcal{E}$ (or $\Delta \mathcal{O}_{G}$ ) that induces a map $\underline{X}(G / B[H]) \rightarrow \underline{X}(G / B[K])$. By the observation, this induces a map

$$
\begin{aligned}
\Psi \underline{X}(G / K[H]) & =\operatorname{Hocolim}_{\Delta \mathcal{E}_{G / K[H]}} \underline{X} \rightarrow \operatorname{Hocolim}_{\Delta \mathcal{E}_{K}} \underline{X} \\
& =I L \underline{X}(G / K[H]) .
\end{aligned}
$$


These maps assemble into the natural transformation $\alpha: \Psi \rightarrow I L$.

Finally, all that remains is to prove the following theorem.

Theorem 7.5. When $\underline{X}$ is in $\operatorname{Im}^{\Delta}, \operatorname{Im}_{0}^{\Delta}$, or $\operatorname{Im}_{p}^{\Delta}$ the natural transformation $\alpha$ is a weak equivalence, rational equivalence, or $p$-adic equivalence respectively.

We begin with a few reductions. Recall that $\underline{X}$ in $\operatorname{Im}^{\Delta}, \operatorname{Im}_{0}^{\Delta}$, or $I m_{p}^{\Delta}$ means that each map $\underline{X}(G / K[H]) \rightarrow \underline{X}(G / K[K])$ is a weak equivalence, rational equivalence, or $p$-adic equivalence for all $H \subseteq K$ respectively. It then follows that $\Psi \underline{X}(G / K[H]) \rightarrow \Psi \underline{X}(G / K[K])$ is also a weak equivalence, rational equivalence, or $p$-adic equivalence since these are homotopy equivalent to $\underline{X}(G / K[H])$ and $\underline{X}(G / K[K])$. Thus, it is enough to show that the map

$$
\Psi \underline{X}(G / K[K]) \rightarrow I L \underline{X}(G / K[K])=L \underline{X}(G / K)
$$

is a weak equivalence, rational equivalence, or $p$-adic equivalence. Moreover, we see from the argument above that the inclusion of $\underline{X}(G / K[K])$ in $\Psi \underline{X}(G / K[K])$ at the zero simplicial level (as the value of $\underline{X}$ on the object $G / K[K])$ is a homotopy equivalence. The map $\alpha$ carries this copy of $\underline{X}(G / K[K])$ to a corresponding copy in $L \underline{X}(G / K)$ at the zero simplicial level. The theorem now becomes an immediate consequence of the following lemma.

Lemma 7.6. Let $\underline{X}$ be in $\operatorname{Im}^{\Delta}, \operatorname{Im}_{0}^{\Delta}$, or $\operatorname{Im}_{p}^{\Delta}$. The inclusion of $\underline{X}(G / K[K])$ in $L \underline{X}(G / K)$ is a weak equivalence, rational equivalence, or $p$-adic equivalence respectively.

Proof. We argue by induction. Let $\mathcal{C}$ be the set of finite collections $C$ of closed subgroups of $G$ such that $K \in C$ and $K \subseteq J$ for each $J \in C$; partially order $\mathcal{C}$ by inclusion. Note that the single element collection $\{K\}$ is the smallest element of $\mathcal{C}$. For any collection $C$ in $\mathcal{C}$, let $\Delta \mathcal{E}_{C}$ be the full subcategory of $\Delta \mathcal{E}_{K}$ with objects $G / B[A]$ where both $A$ and $B$ are in $C$. Let $\Lambda C=$ Hocolim $_{\Delta \mathcal{E}_{C}} \underline{X}$. Observe that $\Lambda$ is a functor on $\mathcal{C}$, since $C \subset D$ induces a map $\Lambda C \rightarrow \Lambda D$. Then $L \underline{X}(G / K)=\operatorname{Colim}_{\mathcal{C}} \Lambda$, and the maps in the colimit system are induced by maps of simplicial spaces which are just inclusions of disjoint summands in each simplicial degree. Therefore, if we can show that the inclusion $\underline{X}(G / K[K])$ in each $\Lambda C$ is an equivalence, passing to the colimit system, we will have shown that the inclusion in $L \underline{X}(G / K)$ is an equivalence.

We induct on the number of elements of $C$. If $C$ has only one element, then $C=\{K\}$ and $\Lambda C=\underline{X}(G / K[K])$. For the inductive step, let $M$ be a maximal subgroup in $C$, so that if $K \subseteq M \subseteq N$ then $M=N$. Let $C_{\widehat{M}}$ be the complement of $\{M\}$ in $C$. When $C \neq\{K\}$, we must have 
$M \neq K$, and we can assume by induction that the inclusion of $\underline{X}(G / K[K])$ in $\Lambda C_{\widehat{M}}$ is an equivalence. Let $\Delta \mathcal{E}_{C ; G \widehat{T M[M]}}$ be the full subcategory of $\Delta \mathcal{E}_{C}$ consisting of all objects except $G / M[M]$. Let $\mathcal{M}$ be the full subcategory of $\Delta \mathcal{E}_{C}$ with objects $G / M[A]$ such that $A \in C$, and let $\mathcal{M}_{G \widehat{/ M[M]}}$ be the full subcategory of $\mathcal{M}$ consisting of all objects except $G / M[M]$. Observe that the only objects of $\Delta \mathcal{E}_{C}$ which have a map to or from $G / M[M]$ are in $\mathcal{M}$, and so

$$
\begin{aligned}
& \operatorname{Hocolim}_{\Delta \mathcal{E}_{C}} \underline{X}
\end{aligned}
$$

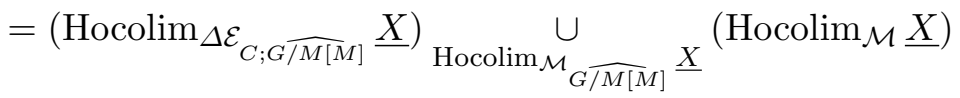

This is a pushout along a cofibration, and so we just need to show that the inclusion of $\Lambda C_{\widehat{M}}$ in $\operatorname{Hocolim}_{\Delta \mathcal{E}_{C ; G / M[M]}} \underline{X}$ and the inclusion of Hocolim $_{\mathcal{M}_{G / M[M]}} \underline{X}$ in Hocolim $\mathcal{M}^{X}$ are equivalences.

To show the first, we show that the inclusion of $\Delta \mathcal{E}_{C_{\widehat{M}}}$ in $\Delta \mathcal{E}_{C ; G / \widehat{M[M}]}$ is left cofinal and invoke Lemma 7.3. Let $G / P[J] \in \Delta \mathcal{E}_{C ; G / M[M]}$, and consider the category incl $\downarrow G / P[J]$. If $P \neq M$ then $G / P[J] \in \Delta \mathcal{E}_{C_{\widehat{M}}}$ and $(G / P[J]$, id) is a final object, so the nerve of this category is contractible. If $P=M$, then $J \subseteq M, J \neq M$ and $(G / J[J], G / J[J] \rightarrow G / M[J])$ is an initial object; again the nerve of this category is contractible.

For the second inclusion, note that $G / M[K]$ is the final object of $\mathcal{M}_{G \widehat{G M[M]}}$. Therefore the nerve of $\mathcal{M}_{G \widehat{/ M[M]}}$ is contractible and the map

$$
\begin{aligned}
\underline{X}(G / M[K]) \rightarrow \underline{X}(G / M[K]) \times & N \mathcal{M}_{G / \widehat{T[M}]} \\
& =\operatorname{Hocolim}_{\mathcal{M}_{G / M[M]}} \underline{X}(G / M[K])
\end{aligned}
$$

is a homotopy equivalence. Now $\underline{X}$ is in $\operatorname{Im}^{\Delta}$, and so all the maps in $\mathcal{M}_{G \widehat{/ M[M]}}$ induce equivalences on $\underline{X}$. Thus,

$$
\operatorname{Hocolim}_{\mathcal{M}_{G / M[M]}} \underline{X}(G / M[K]) \rightarrow \operatorname{Hocolim}_{\mathcal{M}_{G / M[M]}} \underline{X}
$$

is the geometric realization of a simplicial (weak, rational, or $p$-adic) equivalence, and hence an equivalence. The inclusion $\underline{X}(G / M[K])$ in Hocolim $_{\mathcal{M}_{G / M[M]}} \underline{X}$ is therefore an equivalence. On the other hand, $\mathcal{M}$ has an initial object $G / M[M]$, so the inclusion of $\underline{X}(G / M[M])$ in $\operatorname{Hocolim}_{\mathcal{M}} \underline{X}$ is a homotopy equivalence. Furthermore the composite map

$$
\underline{X}(G / M[K]) \rightarrow \underline{X}(G / M[M]) \rightarrow \operatorname{Hocolim}_{\mathcal{M}} \underline{X}
$$

is homotopic to the inclusion of $\underline{X}(G / M[K])$ in $\operatorname{Hocolim}_{\mathcal{M}} \underline{X}$. Thus, the inclusion Hocolim ${ }_{\mathcal{M}_{G / M[M]}} \underline{X}$ in Hocolim $\underline{\mathcal{M}}_{\underline{X}}$ is a weak, rational, or $p$-adic equivalence as required. 


\section{References}

1. A.K. Bousfield and V.K.A.M. Gugenheim, On PL de Rham Theory and Rational Homotopy Type, Mem. Amer. Math. Soc. vol. 8 no 179 (1976)

2. A. K. Bousfield and D. Kan, Homotopy Limits, Completions and Localizations, Lecture Notes in Math. 304 (1972)

3. W. G. Dwyer, D. M. Kan, Reducing Equivariant Homotopy Theory to the Theory of Fibrations, Conference on algebraic topology in honor of Peter Hilton (Saint John's, Nfld., 1983), Contemp. Math. 37, Amer. Math. Soc. pp 35-49 (1985)

4. S. Eilenberg, J. C. Moore, Homology and Fibrations I, Comm. Math. Helv. 40, 199-236 (1966)

5. A. D. Elmendorf, Systems of Fixed Point Sets, Trans. AMS. 277, 275-284 (1983)

6. M. A. Mandell, $E_{\infty}$ Algebras and $p$-Adic Homotopy Theory, Topology, to appear

7. M. A. Mandell, Equivariant $p$-Adic Homotopy Theory, preprint (2000)

8. D. G. Quillen, Homotopical Algebra, Springer Lecture Notes 43 (1967)

9. L. Scull, Rational $S^{1}$-Equivariant Homotopy Theory, Trans. Amer. Math. Soc., to appear

10. D. Sullivan, Infinitesimal Computations in Topology, Inst. Hautes Etudes Sci. Publ. Math. 47, 269-332 (1977)

11. G. Triantafillou, Equivariant Minimal Models, Trans. Amer. Math. Soc. 274, 509-532 (1982) 